\section{EL «PARADIGMA LEDESMA- KOLVENBACH»: ORIGEN Y CONCRECIÓN EN EL ÁMBITO UNIVERSITARIO}

\author{
Aurelio Villa Sánchez \\ Universidad de Deusto \\ aurelio.villa@deusto.es \\ Carl Antonius Lemke Duque \\ Universidad de Deusto \\ carl.antonius.lemke@deusto.es
}

Cómo citar este artículo/Citation: Villa Sánchez, A. y Lemke Duque, C. A. (2016). El "paradigma Ledesma-Kolvenbach»: Origen y concreción en el ámbito universitario. Arbor, 192 (782): a358. doi: http://dx.doi.org/10.3989/arbor.2016.782n6003

Recibido: 04 noviembre 2014. Aceptado: 25 agosto 2015.

RESUMEN: El artículo profundiza en el principio de Educación Superior conocido como "paradigma Ledesma-Kolvenbach», que desde los años más recientes ha orientado la identidad y misión de las universidades jesuíticas de todo el mundo. El paradigma distingue cuatro dimensiones interrelacionadas: la humanitas, la iustitia, la fides y la utilitas. Mediante análisis de contenido cualitativo y análisis de discurso el artículo analiza, primero, el origen y la génesis del modelo a partir de los discursos universitarios de Peter-Hans Kolvenbach (1928-2016), que fue superior general de la Compañía de Jesús desde 1983 hasta 2008. En segundo lugar, el artículo presenta el traspase del paradigma a un modelo valórico cuatrodimensional y una serie de aplicaciones prácticas. Los resultados de ambas partes de la investigación demuestran que el "paradigma Ledesma-Kolvenbach" significa un impulso importante de innovación pedagógica, no obstante de conservar ciertos rasgos de tradicionalismo.

PALABRAS CLAVE: Educación superior; Societas lesu; PeterHans Kolvenbach; análisis de discursos; pedadogía empírica.

\section{THE «LEDESMA-KOLVENBACH PARADIGM॥: ORIGIN AND REALIZATION AT UNIVERSITY LEVEL}

Copyright: (C) 2016 CSIC. Este es un artículo de acceso abierto distribuido bajo los términos de la licencia Creative Commons Attribution (CC BY) España 3.0.
ABSTRACT: This paper deals with the higher educational principle known as the "Ledesma-Kolvenbach paradigm», which has served as a guideline for the identity and mission of Jesuit universities worldwide over the past few years. The paradigm covers four interrelated dimensions: humanitas, iustitia, fides and iustitia. Using qualitative content analysis and discourse analysis, the paper delves, firstly, into the origin and development of the paradigm, analyzing the academic lectures of Peter-Hans Kolvenbach (1928-), who presided as Superior General of the Society of Jesus between 1983 and 2008. Next, it addresses the transformation of the paradigm into a fourdimensional value model, followed by a number of practical applications in academia. Finally, the paper summarizes how the "Ledesma-Kolvenbach paradigm» constitutes a significant step forward in practical educational innovation in spite of retaining remnants of premodern tradition.

KEYWORDS: Higher Education; Societas lesu; Peter-Hans Kolvenbach; John Henry Newman; Discourse Analysis; Empirical Pedagogy. 
"Un documento que describe las características de la educación de la Compañia no es una nueva Ratio Studiorum. Sin embargo, del mismo modo que la $R a-$ tio nacida a finales del siglo XVI, y como continuación de la tradición que entonces empezó, este documento puede darnos a todos una visión común y un común sentido de nuestra finalidad; puede ser tambien un modelo con el que nos contrastemos a nosotros mismos." (Kolvenbach, 1986/1992, p. 143).

\section{INTRODUCCIÓN}

El así llamado «paradigma universitario LedesmaKolvenbach» es una re-titulación hecha por parte de la literatura científica en 2008 con respecto a las reflexiones del P. General de la Compañía de Jesus Peter-Hans Kolvenbach (1928-) sobre temas de educación jesuita y enseñanza superior en centros universitarios y académicos de la orden (Agúndez Agúndez, 2008a, pp. 630-631; Agúndez Agúndez 2008b, pp. 24-26). El re-titulado "paradigma» de Kolvenbach fue recibido y expuesto enseguida por el P. General sucesor Adolfo Nicolás Pachón (1936-) en su conferencia en la ESADE en Barcelona Misión y Universidad. ¿Qué futuro queremos? (2008), aunque dejó de tener relevancia en sus contribuciones ulteriores sobre educación jesuita a partir del 2010 (Nicolás Pachón, 2008; Nicolás Pachón 2010a; Nicolás Pachón 2010b; Nicolás Pachón 2011; Nicolás Pachón 2013a; Nicolás Pachón 2013b; Nicolás Pachón 2013c; Nicolás Pachón 2013d). Además, las re-tituladas reflexiones pedagógicas de Kolvenbach han sido comentadas y discutidas, de forma extensiva, en el ámbito académico enfocando particularmente el horizonte educativo europeo (Guibert, 2009, pp. 161163; Oráa, 2010, p. 14).

El propio Kolvenbach, no obstante, al presentar las Características de la Educación de la Compañía de Jesus elaboradas por la Comisión Nacional de Educación SJ (CONED), ya había hablado de un "modelo", en el sentido de una orientación básica: capaz tanto de dar continuidad como de renovar la Ratio Studiorum, presentada por el P. General Claudio Acquaviva (1543-1615) en Roma en 1586 no "como algo definitivo y terminado, porque eso sería muy difícil y probablemente imposible, sino más bien como un instrumento que nos ayudará a afrontar cualquier tipo de dificultades que podamos encontrar, ya que él proporciona a toda la Compañía una perspectiva unitaria" (Kolvenbach, 1986/1992, p. 145). Además, en 1993, la Compañía de Jesus presentó su más reciente documento oficial básico de educación como fruto del Consejo Internacional de la Educación SJ (ICAJE) que hace referencia directa al trabajo de la CONED presentado 400 años después de la Ratio, diseñado como "desarrollo práctico de las "Características»". Sobre la base del discurso de Kolvenbach $L a$ Pedagogía Ignaciana Hoy (1993/2008, pp. 124-139), estas orientaciones prácticas se refieren de forma explícita y extensiva a un "Paradigma Pedagógico Ignaciano" (Duminuco, Prólogo a Pedagogía ignaciana. Un planteamiento práctico). Finalmente, Kolvenbach concretó, en cuatro discursos entre 2001 y 2007, con referencia directa al teólogo español y co-autor de la Ratio Diego de Ledesma (1519-1575), los cuatro conceptos elementales para la enseñanza jesuita (utilitas, iustitia, humanitas, fides) que en 2008 se re-titularon como "paradigma Ledesma-Kolvenbach» (Kolvenbach, 2001/2008; Kolvenbach, 2006/2008a; Kolvenbach, 2006/2008b; Kolvenbach, 2007/2008).

Aunque este trasfondo explica los motivos de la retitulación posterior, el enfoque final en Ledesma no fue algo original de Kolvenbach. De hecho, los cuatro principios generales de educación ledesmanianos fueron presentados ya como referente explícito para la pedagogía jesuita, por ejemplo, en la introducción al primer número de las Conversations on Jesuit Higher Education en 1992 citando la versión traducida de la Monumenta Pedagogica Societatis lesu (MPSI, 1586) en el estudio The Jesuit Code of Liberal Education. Development and Scope of the Ratio Studiorum (1938) del jesuita estadounidense Allan Peter Farrell (1896-s.e.) (Padberg, 1992, p. 3). En el primer libro De Ratione et Ordine Studiorum in Collegii Romani integrada en la MPSI, Ledesma había expuesto los cuatro ingredientes fundamentales de la educación, es decir, (1) el «Conocimiento y Capacidad necesaria para una carrera productiva», (2) la "Responsabilidad social y política», (3) la «Contribución en el desarrollo total de la Persona Humana» y, finalmente, (4) la «Fe en Dios» del siguiente modo:

"Scholae literariae hominum generi, republica uero xpiana. et Xpi. ecclesia, maxime sunt necessariae, tum ad multas uitae huius commoditates, tum ad rectam rerum publicarum gubernationem et leges, tum ad naturae ipsius rationalis ornatum et splendorem ac perfectionem, tum demum, quod maius est, ad Dei fidem et religionem docendam, defendendam et propagandam; et ut homines ad suum tandem finem ultimum commodius et facilius perducantur: de quibus primo dicendum quae et quales sint, et in quo differant" (Gómez Rodeles, Lecina, Agustí, Cervos y Ortiz, 1901, p. 345). 
Dentro de la amplia investigación internacional sobre la historia de la pedagogía jesuita (Blanco Trías, 1922; Malaxechevarría, 1926; Donelly, 1934; de Dainville, 1940; Charmot, 1952; Donohue, 1963; Dignath, 1977; Bailey, 1978; Hughes, 1981; Labrador Herraiz, 1987; Carmody, 1992; García Iglesias, 1994; García Iglesias, 1995; Azcarate Ristori, 1996; Revuelta González, 1998; McKevitt, 2008; Whitehead, 2013), sin embargo, el análisis de Ledesma con respecto a la Ratio y la uniformitas doctrinae de la Compañía no ha llegado a tener importancia sistemática (Friedrich, 2009). Comparando su papel con el del "unificador, recopilador y promulgador oficial" de la Ratio Acquaviva, la relevancia de Ledesma es de menor peso y menor influencia en su extensión (Labrador Herraiz, 1999, pp. 33-35 y pp. 40-42; Padberg, 2000, pp. 83-85). Debido a la enorme difusión europea de su Dottrina christiana (ital. 15671571, cast. 1573 etc.), los cuatro principios del catecismo de Ledesma (Fe, Esperanza, Oración y Caridad) han recibido más atención por parte de la investigación (Ledesma, 1611, pp. 10-12, pp. 30-32, pp. 43-45 y pp. 66-68; Resines Llorente, 1997, pp. 259-261).

Para hacer transparente el origen evolutivo del "paradigma Ledesma-Kolvenbach» y su concreción en el ámbito universitario, las siguientes páginas analizarán, primero (I.), los textos claves de Kolvenbach sobre pedagogía jesuita 1985-2007 y, segundo (II.), la traslación del paradigma a un Modelo Valórico Cuatridimensional con su respectiva aplicación concreta en el ámbito universitario. En un resumen final se contrastarán, finalmente, los resultados expuestos.

\section{PEDAGOGÍA JESUITA SEGÚN KOLVENBACH 1985-2007}

A parte de las intervenciones más ocasionales en torno a la educación y enseñanza superior jesuita (Kolvenbach, 1994/2008), las numerosas reflexiones de Kolvenbach sobre materias de la pedagogía se pueden diferenciar en dos fases cronológicas que corresponden con dos accesos sistemáticos y complementarios: (A) Una primera fase entre 1985 y 1994 que abarca seis intervenciones centrales (Kolvenbach, 1985/2008; Kolvenbach, 1986/1992; Kolvenbach, 1987/2008; Kolvenbach, 1989/2008; Kolvenbach, 1990/2008; Kolvenbach, 1994/2008) y, (B) una segunda fase entre 2001 y 2007 compuesta por cuatro discursos claves (Kolvenbach, 2001/2008; Kolvenbach, 2006/2008a, 2006/2008b, 2007/2008) ${ }^{1}$.

\section{(A) Pedagogía jesuita según Kolvenbach 1985-1994}

A lo largo de las seis intervenciones tempranas entre 1985 y 1994, el horizonte común de la primera fase de reflexiones pedagógicas de Kolvenbach se plasma en tres enfoques fundamentales desarrollados y compartidos progresivamente con algunas reiteraciones:

En primer lugar (1), Kolvenbach se preocupa por la tensión entre las prioridades como efecto del famoso Decreto 4 (Congregación General 32) sobre la "connaturalidad del jesuita, es decir, su "opción preferencial" por los pobres como "autentica naturaleza apostólica del sector educacional" insistiendo que "no hay conflicto" porque "desde la perspectiva de la promoción de la justicia (...) toda investigación debe promover, en último término, la dignidad de la persona humana" (Kolvenbach, 1985/2008, pp. 34-36 y pp. 38-40).

En segundo lugar (2), Kolvenbach se ocupa extensivamente del tema del concepto de la universidad como "instrumento de apostolado" subrayando su capacidad de auto-renovación mediante la "atención personalizada a cada alumno concreto... y cada profesor concreto" (cura personalis), una "elevada calidad académica" y la "estrecha colaboración con la Iglesia jerárquica" en torno a la evangelización (Kolvenbach, 1985/2008, pp. 41-43; Kolvenbach, 1986/1992, p. 144; Kolvenbach. 1989/2008, pp. 64-66). Según Kolvenbach, el objetivo nuclear de la universidad, sin embargo, consiste en generar y fomentar la "unidad y coherencia" de la ciencia, "»universalizando» las ciencias particulares". El instrumento decisivo para conseguir ese objetivo es una "interdisciplinaridad" capaz de la "integración cualitativa de la investigación que desemboque en una verdad más amplia" con el fin de "explicar la totalidad de la creación" orientada en la "transfiguración" de Cristo, es decir, la "Encarnación humanizadora de Dios" que diviniza al hombre (Kolvenbach, 1987/2008, pp. 51-53; Kolvenbach, 1989/2008, pp. 61-63; Kolvenbach, 1990/2008, pp. 92-94; Kolvenbach, 1994/2008, pp. 149-151).

En tercer lugar (3), Kolvenbach profundiza intensamente en la "realización del hombre integral" por medio de valores comprendidos tridimensionalmente según la espiritualidad ignaciana en (a) la percepción intelectual de lo que tiene valor, (b) su afección emocional y (c) la acción real y concreta motivada por ello. Es aquí, justamente, cuando el fin de la enseñanza jesuita de crear "agentes multiplicadores" capaces de "actuar como levadura en la transformación de las actitudes, humanizando el clima social" se convierte, más explícitamente, en la formación de "los lideres del tercer milenio" (Kolvenbach, 1985/2008, p. 38; Kolvenbach, 1989/2008, pp. 58-60; Kolvenbach, 1990/2008, p. 90). Al hablar, en este contexto, de la "realización del hombre integral", mejor dicho, de 
la "realización integral de la persona humana" (Kolvenbach, 1987/2008, p. 51; Kolvenbach, 1989/2008, p. 63; Kolvenbach, $1994 / 2008$, p. 151), Kolvenbach hace referencia implícita al personalismo cristiano de Max Scheler (1874-1928) y Jacques Maritain (18821973) (Spader, 2002; Schneck, 2002; Ritzler, 2000) introducida en España a través del círculo de discípulos y colaboradores de José Ortega y Gasset (1885-1955) (Lara, 2010; Lemke Duque, 2014, pp. 88-90, pp. 298301, pp. 432-439 у pp. 456-460).

Sin embargo, el hilo conductor más característico de la primera fase de intervenciones es la reiterativa cita directa de Kolvenbach (desde 1987 hasta incluso en su primer discurso de la segunda fase en 2001) al famoso cardenal y teólogo de Oxford Paul Henry Newman (1801-1890), convertido al catolicismo en 1845 y beatificado por Benedicto XVI. en 2010 (Biemer, 2009; Schwanke, 2011; Larrañaga Oyarzabal, 2012). Esas reiteradas referencia a Newman se efectúan, explícitamente, al concepto de universidad que el cardenal inglés había trabajado intensamente en nueve discursos, al ser encargado de la fundación de la Universidad Católica de Dublín, recopilados como The Idea of a University defined and illustrated (1852/1859). Debido a la mediación del cardenal Julius Döpfner (19131976) y otros, Newman es considerado como una de los padres fundadores del Segundo Concilio Vaticano (1962-1965) (Döpfner, 1945) y sigue siendo un referente tanto para la modernización pedagógica como para cambios político-sociales (Beirne, 1996, pp. 3444, p. 66 y p. 240; Buckley, 1998, pp. 61-63, p. 123 y p. 144). En sus nueve discursos sobre The Idea of a University ofrece un acceso liberal al tema de la educación y enseñanza superior que se plasma, sobre todo, en (a) su enfoque en "teaching universal knowledge" (b) sin necesidad de la "authority of the Church, or any authority at all" y (c) sin reducción a "Christian Literature" únicamente para profesar "all branches of knowledge" con el fin de (d) "prepare for the world", es decir, "fit men of the world for the world" (Newman, $1885 / 1907$, p. viii, p. 7, p. 20 , p. 166, p. 229 y p. 232). Newman, además, critica de forma directa la reducción utilitarista de la educación insistiendo en una «educación liberal» (Liberal Education) cuya "utilidad» (usefullness/utility) se centra en fomentar las capacidades individuales del estudiante (Newman, 1885/1907, pp. 151-179).

\footnotetext{
"This process of training, by which the intellect, instead of being formed or sacrificed to some particular or accidental purpose, some specific trade or profession, or study or science, is disciplined for its own
}

sake, for the perception of its own proper object, and for its own highest culture, is called Liberal Education [...]. [...] And to set forth the right standard, and to train according to it, and to help forward all students towards it according to their various capacities, this I conceive to be the business of a University" (Newman, 1885/1907, pp. 152-153).

No obstante, la investigación científica también ha insistido, últimamente, en el carácter tradicional conservador que subyace al concepto de educación y enseñanza superior de Newman. Habría que subrayar, sobe todo, que la función de la universidad no consiste, según el cardenal inglés, en aumentar y ampliar la participación social mediante el acceso y la integración académica sino, primordialmente, en el liderazgo político mediante la creación de una minoría directiva cuya preparación como élite esta apoyada en el canon clásico de los valores católicos (Schwanke, 2013, pp. 52-54). Habría que tener en cuenta, además, la ostensible cercanía de Newman a posiciones creacionistas que sigue alimentando un controvertido debate actual (Newmann, 1873; Kalthoff, 2001; Hütter, 2013).

\section{(B) Pedagogía jesuita según Kolvenbach 2001-2007}

Aunque Kolvenbach había hecho referencia, ya en 1989, a la importancia de la Ratio conmemorada en 1986 con la presentación de las Características de la Educación de la Compañía de Jesús de la CONED (Kolvenbach, 1989/2008, p. 68), no menciona explícitamente a Ledesma hasta su discurso en Roma en 2001. Esta intervención es, además, la última con referencia expresa a Newman siendo, a la vez, la que más líneas directas dedica al cardenal inglés. En sus discursos entre 2006 y 2007 todas las referencias explícitas -a parte de las citas contínuas que hace a su antecesor Pedro Arrupe (1907-1991) y a Ignacio de Loyola (1491-1556)remiten a Ledesma. El marco común de la segunda fase de reflexiones pedagógicas de Kolvenbach entre 2001 y 2007 se plasma en dos tendencias:

En primer lugar (1), Kolvenbach expone bajo el lema del "carisma fundacional" ignaciano las "cuatro razones" (utilitas, iustitia, humanitas, fides) de la educación jesuita concretadas por Ledesma como ejes más centrales para toda la enseñanza de la Compañia, citando directamente de las fuentes originales de la MPSI de 1586 (Kolvenbach, 2001/2008, pp. 193-195). Sintomaticamente, esa exposición de la concreción ledesmaniana se inspira explícitamente en la fuerte presencia de los mismos principios fundamentales de educación en el ámbito universitario estadounidense de la Compañía como fruto del Grupo de Trabajo del 
Institut of Jesuit Sources dirigido por John Padberg en la Universidad Georgetown (Kolvenbach, 2007/2008, pp. 259-260). Además, ante el trasfondo de la creciente "secularización y descristianización", Kolvenbach propone las "razones" de Ledesma como "transposición moderna" de la problemática entre la "dimensión académica" y la "dimensión apostólica", es decir entre las "finalidades propias de la universidad" y la "inspiración cristiana e ignaciana" (Kolvenbach, 2001/2008, pp. 195-197), apuntando tres ámbitos de aplicación: (A) «Academia y Sociedad» - exigiendo un reajuste pragmático del "vínculo entre vida académica y sociedad humana" critica abiertamente el concepto de ciencia de Newman limitado a "la capacidad de ser un fin en si mismo"; (B) «Academia y Globalización» - exigiendo una "globalización de la solidaridad", otra vez, critica directamente a la idea de Newman de la "Universalidad del conocimiento" e identifica una serie de problemas reales del mundo actual como, por ejemplo, la crisis de la democracia, el neocolonialismo, la violencia de los derechos humanos etc. Aquí, la argumentación desemboca en la necesidad de un diálogo intercultural e interreligioso (Kolvenbach, 2001/2008, pp. 199-202) que, obviamente, se inspira en la idea de afrontar, de modo más concreto, los retos de la postmodernidad trabajados intensamente, sobre todo desde principios de los años noventa, por parte de los jesuitas en los Estado Unidos (Byrnes, 1992; Rehg, 2006, pp. 201-208 y pp. 213-215); (C) «Academia y Mercado" - exige en contra del "peligro del elitismo" perseguir la "verdad total" frente a una "verdad estrecha" impuesta por el "factor económico" haciendo la primera y única vez referencia asociativa a Newman (Kolvenbach, 2001/2008, pp. 203-204). Finalmente, el discurso de Kolvenbach del 2001 expone detalladamente la necesaria colaboración de laicos y cooperación interuniversitaria con lo cual subraya el origen y carácter histórico de la enseñanza jesuita, en contra de un concepto "cerrado" y a favor de una universidad abierta "a las personas y tiempos" (Kolvenbach, 2001/2008, pp. 204-208).

En segundo lugar (2), Kolvenbach insiste expresamente en la "armonía entre el alcance educativo de Diego de Ledesma y los programas de una universidad moderna de pedagogía ignaciana" repitiendo el carácter "práctico y social, humanista y religioso" de los "cuatro motivos" ledesmanianos (utilitas, iustitia, humanitas, fides) para proclamar un "nuevo diálogo entre fe y ciencia", entendido en el sentido de un "diálogo siempre a rehacer", como tarea principal de Universidad que, a la vez, debe "integrarse en el Espacio Europeo" Superior de Educación (EEES) "a través de la puesta en practica de la Declaración de Bolonia" (Kolvenbach, 2006/2008a, pp. 240-241 y pp. 246-247; Kolvenbach, 2006/2008b, p. 250).

\section{DEL «PARADIGMA LEDESMA-KOLVENBACH» A LA TRASLACIÓN A UN MODELO VALÓRICO CUATRIDI- MENSIONAL}

Entre 2000 y 2010 se propuso por parte del vicerrector de innovación y calidad, responsable de la formación en la Universidad de Deusto, un marco operativo en el que pudieran trabajarse los valores educativos desde un punto de vista unitario e integral. El modelo que se describe a continuación fue planteado y experimentado en la Universidad de Deusto, intentando implicar en el desarrollo del mismo a instancias de muy distinto nivel. En realidad, lo que se pretendió hacer fue hacer más operativo el desarrollo de los valores en la Universidad de Deusto (UD) enfatizando que aunque los valores pueden y deben promoverse con distintas actividades y desde muchos ángulos, todos ellos forman un conjunto unitario en el quehacer universitario (Consortium of Institutions for Development and Research in Education in Europe, 1991a, Consortium of Institutions for Development and Research in Education in Europe, 1991b; Bunes et al., 1993; Organization for Economic Co-operation and Development, 1993; Muñoz-Repiso, 1994, p. 352; Schwartz, 2001, p. 55; Eléxpuru Albizuri y Medrano Samaniego, 2002; Eléxpuru y Bolívar Botía, 2004). Y esto, de algún modo, se pudo observar cuando por primera vez se invitó a todas las personas o representantes de las diferentes unidades que trabajan valores desde sus puestos académicos bien de docentes, de investigación, de servicios o de extensión. Y a partir de esta reunión, se propuso incorporar cuatro planos que ayudasen a comprender esta unidad de acción divergente en sus acciones y al mismo tiempo convergente en su finalidad. Se formó una comisión ad hoc, coordinada por el vicerrector de Identidad y Misión, Javier Pardo S.J., y por el vicerrector de innovación y calidad, Aurelio ViIla. La Universidad de Deusto tenía un análisis de los valores contenidos en sus documentos institucionales en los que aparecían casi 40 valores (Hall, 1997), a pesar de que ciertas investigaciones apuntasen a que "las personas no pueden concentrarse en más de tres o cuatro valores que realmente produzcan impacto sobre el comportamiento" (Blanchard y colaboradores, 2007, p. 67). Por esto último, dicha comisión de la Universidad de Duesto realizó una tarea de seleccionar y redefinir los valores teniendo en cuenta las cuatro dimensiones del "paradigma Ledesma-Kolvenbach», que finalmente se redujeron a 12 , tres vinculados a 
cada una de las cuatro dimensiones como puede apreciarse en la Figura 1, que posteriormente fueron ratificados por el Consejo de Gobierno de la Universidad de Deusto y el rectorado en el Plan Estratégico de 2010.

(A) UTILITAS - Dimensión práctica: La Universidad se propone formar para la excelencia profesional a través de la excelencia académica, asentando a ambas en los valores morales y en la dinámica del magis. (1) Orientación al conocimiento: Disposición a través del aprendizaje abierto al saber y a la investigación, de una adecuada comprensión de la realidad sustentada en la verdad. (2) Innovación y creatividad: Generación de ideas, elaboración y expresión de nuevas propuestas o adecuaciones de propuestas existentes a nuevos contextos, teniendo en cuenta su viabilidad, convirtiéndolas en realidades prácticas y socialmente valiosas. (3) Visión Global: Percepción de una imagen de la realidad sistémica, universal y holística -más allá de la parcialidad, el particularismo y el individualismo-, inspirada en una valoración moral, y ser capaz de comunicarla.

(B) IUSTITIA - Dimensión social: Persigue formar personas "para los demás y con los demás», comprometidas, en cuanto ciudadanos, con la construcción de estructuras sociales, económicas y políticas justas, desde el dinamismo de la "opción preferencial por los pobres». (4) Igualdad: Defensa coherente de que todos los seres humanos tienen todos los derechos inherentes a su dignidad -y sus correspondientes deberes-, al tiempo que se reconocen sus diferencias y especificidades. (5) Cultura para la Justicia: Actuación para abordar, confrontar y corregir condiciones de desigualdad y opresión humana, lo que supone una redistribución de los recursos, con el fin de hacer efectivo el hecho de que todo ser humano tiene el mismo valor. (6) Compromiso social: Utilización de las propias cualidades y destrezas para realizar una contribución positiva a la sociedad, en especial afrontando las desigualdades, opresiones y marginaciones, a través de la participación cívica, el trabajo profesional y el voluntariado.

(C) HUMANITAS - Dimensión humanista: Se propone estimular la formación de la "persona integral», sustentada moralmente en la centralidad de nuestra común dignidad. Por lo que respecta a la vertiente intelectual, que no debe ser exclusiva, fomenta el cultivo de la racionalidad en su complejidad, la que, en el respeto a su autonomía, no se cierra a la fe religiosa. (7) Desarrollo Personal: Cultivo y manifestación de la totalidad integrada de las capacidades en uno mismo y en los demás. (8) Dignidad Humana: Reconocimiento y respeto en la práctica que todo ser humano es en sí mismo valioso, no instrumentalizable, capaz de iniciativa autónoma; lo que le da derecho a desarrollar satisfactoriamente sus capacidades, de manera que puede desarrollar plenamente su potencial. (9) Responsabilidad ética: Actuación de acuerdo con principios morales fundados, discerniendo prudencialmente su aplicación a la realidad, de modo tal que, cumpliendo las exigencias de la justicia que incluye deberes ante los demás y ante sí mismo, se asuman las consecuencias previsibles de las decisiones tomadas.

(D) FIDES - Dimensión religiosa: Invita a los estudiantes a procesos de búsqueda del sentido último de la vida. Siendo empáticamente sensible a la pluralidad ante lo religioso, ofrece en concreto el horizonte fundante e integrador de la fe cristiana, el que se expresa en el encuentro personal con el Jesús del evangelio. (10) Búsqueda de sentido: Apertura al descubrimiento de un significado profundo de la propia existencia y de la realidad en su conjunto. (11) Diálogo interreligioso: Orientación a las propias convicciones a la intercomunicación con personas o grupos de otras creencias religiosas desde el respeto y en condiciones de equidad. (12) Fe Cristiana: Apertura experiencial al Misterio del Dios revelado en Jesucristo, que estimula al seguimiento de su persona.

Puede verse en la Figura 1, el modelo de las cuatro dimensiones que desde nuestro punto de vista se ha señalado con la metáfora de la hélice, en el sentido que es la que en su movimiento es capaz de mantener el motor (los valores) en marcha. Como muy bien describe el P. Agúndez (2008a) cuando se refiere al modelo presentado:

"Las cuatro aspas de la hélice se mueven al tiempo sobre un mismo y único eje (I+M) (se refiere a la I de identidad, y a la $\mathrm{M}$ de Misión) que significa que, en las finalidades pedagógicas de la enseñanza jesuítica, no es comprensible la utilitas (competencia práctica, excelencia profesional) sin la iustitia (compromiso social); ni aquella y ésta sin la humanitas (maduración humana, formación de la "persona integral», de la «persona toda") y ninguna de las tres sin la fides (el anclaje dinámico en una opción radical de sentido de la persona y de la vida, que es al mismo tiempo directriz básica del comportamiento ético). Lo mismo vale en lectura inversa: la dimensión radical de sentido alimenta, moviliza y potencia el soporte humano que, con responsabilidad social, desarrolla su competencia profesional útil en provecho propio y de la sociedad" (Agúndez Agúndez, 2008a, p. 606). 
Figura 1. Modelo de las cuatro dimensiones

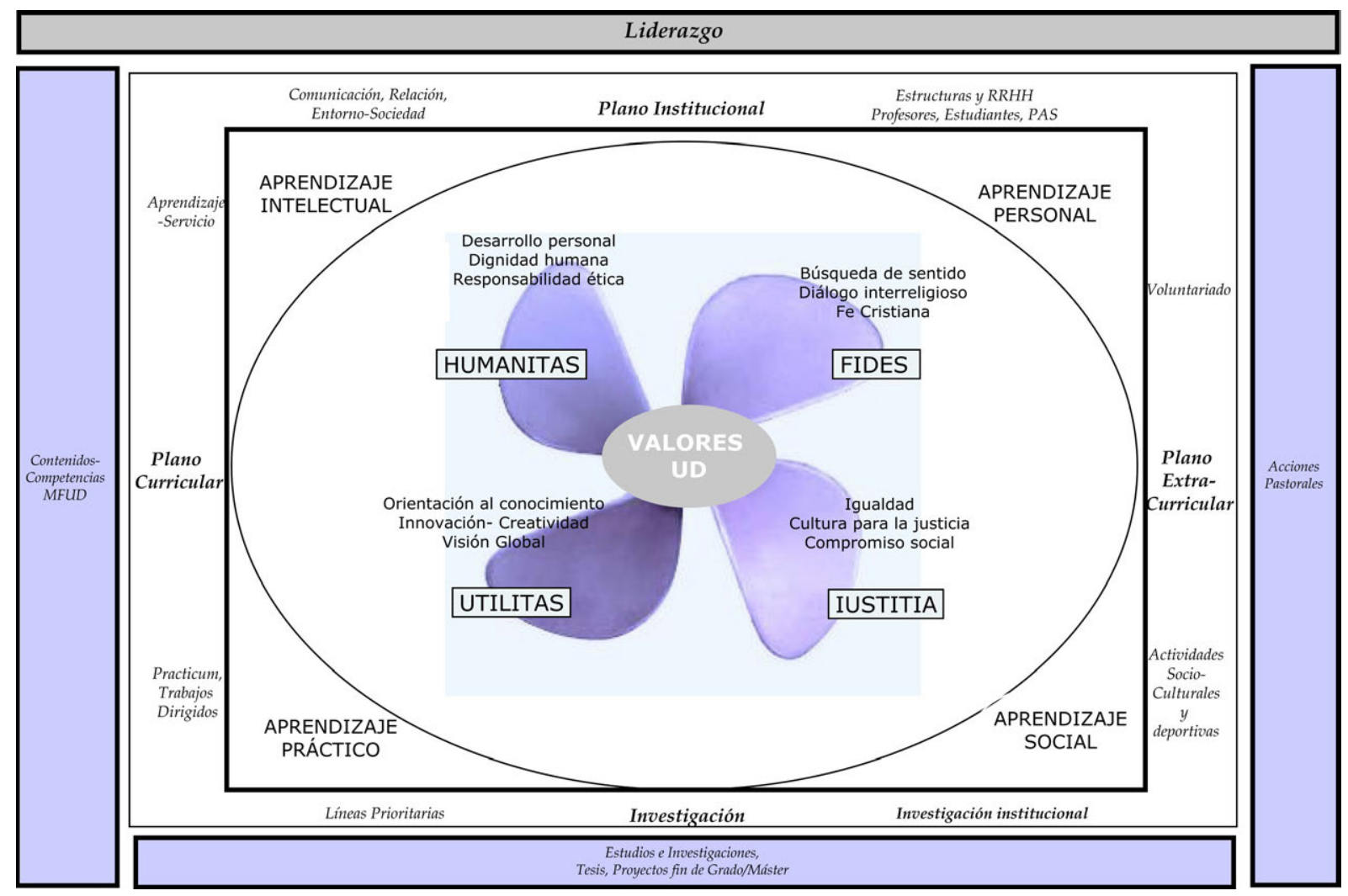

Fuente: Elaboración propia

El modelo se aplica a cuatro grandes planos del quehacer universitario: (I) el plano institucional, (II) el plano curricular, (III) el plano extra-curricular y (IV) el plano de la investigación. Ciertamente quedan muchos planos por desarrollar, pero estos cuatro planos fueron los definidos como los clave en una primera aplicación de los valores.

\section{(I) Primer plano: Liderazgo}

El primer plano representa la clave institucional que hemos concretado en tres aspectos: el liderazgo ejercido por los responsables directivos universitarios desde su máxima figura como es el rector, hasta el último profesor, pasando por todos los niveles de responsabilidad directiva: vicerrectores, decanos, vicedecanos, secretarios de facultad, directores de departamento o cualquier otro cargo que tenga bajo su dirección personal que coordinar o dirigir:

(I-A) Para desarrollar este liderazgo es necesario en primer lugar definir el tipo de liderazgo que se desea promover, entendiendo por liderazgo la capacidad de influencia que una persona tiene para lograr alcanzar los resultados esperados implicando a las personas que están a su cargo. Tres aspectos son clave para el liderazgo: la misión de la institución, la visión y los valores. Estos conceptos, manejados en la actualidad en todas las organizaciones, constituyen en la universidad un aspecto clave. Como se ha indicado por parte de la investigación se podría entender la visión como la misión más los valores (McDermott y O'Connor, 1999, p. 143). El liderazgo debería por tanto recoger la misión de la universidad y sus valores principales, y esto es lo que se intentó realizar, al definir el estilo de liderazgo como liderazgo ignaciano. Por otra parte, se requiera formar al personal. Definido el liderazgo ignaciano como un tipo de liderazgo basado en el servicio a los demás, en la promoción de los valores de la Fe y la Justicia. Se han organizado durante los últimos años, sesiones de formación sobre el tema. Recientemente, se ha organizado en la Universidad de Deusto un seminario sobre esta temática con la participación de estudiosos jesuitas, especialmente latinoamericanos (Seminario sobre Liderazgo Ignaciano y Justicia Social). Este aspecto está tratado en este mismo nú- 
mero por el actual Rector de la Universidad de Deusto (Guibert, 2014; Lowney, 2005).

(I-B) Un segundo aspecto importante es lo que hemos denominado: Estructuras y Recursos humanos, es decir, que las políticas institucionales estén embebidas de los valores que se desean potenciar lo que ofrecerá, además de transparencia, la confianza y credibilidad entre las personas que conviven en la universidad. Las estructuras organizativas conforman el estilo de actuación que se pretende instalar en el procedimiento y trato con el personal. Posiblemente, no hay peor política para cualquier institución que definir unos valores y que éstos no se reflejen en sus modos de actuación. En este aspecto, se destaca la elaboración de una herramienta para el diagnóstico de la aplicación de valores cuyo propósito es ayudar a diagnosticar el desarrollo de los valores incluidos en los ámbitos UTILITAS, IUSTITIA, HUMANITAS y FIDES en los diferentes planos institucionales de la Universidad de Deusto, atendiendo a los impactos que ésta genera tanto en el seno de su organización como en el resto de la sociedad. Esta herramienta fue desarrollada desde el Aula de Ética (Promoción del desarrollo de valores en los diferentes planos institucionales de la Universidad de Deusto. Herramienta para su diagnóstico. [Documento inédito]).

Esta herramienta de diagnóstico de la aplicación de los valores que la Universidad había definido, trata diferentes ámbitos: Educativo, organizacional, cognitivo, y social, está destinada para ser usada por el rectorado (rector y vicerrectores). Tiene un enfoque de autodiagnóstico en el que los máximos responsables evalúan 196 cuestiones, lógicamente cada responsable en su área. La propuesta de la herramienta es valorar con una puntuación cada elemento en una escala de 1 a 10 , y posteriormente incorporar evidencias que avalen esa puntuación. Este diagnóstico permite un acercamiento al desarrollo institucional de los valores y comprobar el nivel alcanzado, lo que conlleva la posibilidad de mejora y modificaciones de las estrategias.

(I-C) El tercer elemento de este plano institucional, viene conformado por la Comunicación y relación con la Sociedad. Es muy importante que la sociedad perciba a la universidad a través de los valores que desea transmitir y que exista una congruencia entre la imagen y perfil institucional deseado con el percibido, lo que no ocurre en todos los casos. El sociólogo Francisco Garmendia realizó una investigación para examinar la imagen que la Universidad de Deusto tenía en los medios de comunicación impresos y digitales en comparación con las otras universidades del entor- no, con el propósito de recoger datos para estudiar la congruencia entre los valores promovidos o deseados por la Universidad de Deusto y los que realmente son percibidos en el entorno social (como indica Garmendia en un documento inédito titulado Estudio sobre los valores que identifican dominantemente a la $U$ [niversidad de] D[eusto] en prensa e Internet).

\section{(II) Segundo plano: Curricular}

El segundo plano es el Curricular y pedagógico, que se ha subdivido en tres elementos: el contenido-competencias a desarrollar en el perfil académico-profesional de los estudiantes; el aprendizaje-servicio, y finalmente, el practicum y trabajos dirigidos:

(II-A) La selección de las competencias a desarrollar constituye un primer paso importante para el desarrollo de los valores, porque a través de las mismas se pueden potenciar actitudes y valores. Por ejemplo, se pueden seleccionar competencias como Trabajo en Equipo, Comportamiento ético, Comunicación Interpersonal, entre otras que favorezcan la disposición hacia los demás y su valoración. A través de los contenidos o temas, se puede sensibilizar a los estudiantes en los problemas que existen tanto en el contexto cercano como en los contextos nacionales e internacionales en los que las diferencias entre ricos y pobres y a través de estos problemas sensibilizar hacia la solidaridad, el compromiso social, la búsqueda de soluciones más justas y equitativas entre todas las personas y los pueblos.

(II-B) El segundo aspecto hace referencia al aprendizaje-servicio, que es una modalidad que viene desarrollándose en muchos países del mundo para incrementar el compromiso y la responsabilidad social universitaria en profesores y estudiantes con el entorno en el que viven. El Higher Education Quality Committee define el aprendizaje-servicio del siguiente modo: "Aprendizaje aplicado que se dirige a las necesidades específicas de la comunidad y se integra en el programa y currículum académico. Puede contar con créditos y ser evaluado, y puede o no realizarse en un ambiente laboral" (Higher Education Quality Committee, 2006). La vocación de servicio está claramente presente en la pedagogía ignaciana. De hecho, los procesos de transformación universitaria y comunitaria se formulan en clave de justicia social desde las universidades jesuitas (Combs y Ruggiano, 2013), pudiendo llegar a concretarse en acciones como las derivadas del aprendizaje-servicio (Cuban y Anderson, 2007). A su vez, su misión, fundamentada en el servicio a la fe y la promoción de la justicia, requiere 
una gestión "eficaz, eficiente y de calidad" (Gargantini y Zafforoni, 2009, p. 19).

(II-C) El tercer aspecto del plano curricular era el practicum y trabajos fin de grado o de máster. El propósito de este tercer aspecto consistía en fomentar entre los estudiantes la oportunidad de llevar a cabo su practicum en sus respectivas carreras con un enfoque claramente social, para vivenciar y acercarse a ámbitos sociales desconocidos para muchos de los estudiantes, y que seleccionaran temas y enfoques de sus trabajos fin de carrera o de máster de carácter social, es decir, aplicar los conocimientos desde sus áreas (ingeniería, psicología, educación, humanidades, etc.) en contextos sociales de su entorno o problemas mundiales.

\section{(III) Tercer plano: Extra-Curricular}

El tercer plano se refiere al plano extra-curricular que es el ámbito en el que los estudiantes pueden desarrollar sus capacidades sociales, deportivas o altruistas. Tres aspectos principales configuran este tercer plano: el voluntariado, las actividades deportivas y las actividades pastorales.

(III-A) El voluntariado, como una oferta abierta para aquellos estudiantes que desean compartir su tiempo colaborando en acciones sociales que llevan a cabo distintas ONG o Fundaciones con orientación social. Estas actividades están al margen de las actividades académicas oficiales, de ahí su distinción con el practicum o el aprendizaje-servicio.

(III-B) El segundo aspecto son las actividades pastorales, que ofrecen la oportunidad a los estudiantes de profundizar en su fe, y llevar a cabo actividades diarias para orar juntos, o actividades como «el camino a Santiago», la «javeriada» consistente en la peregrinación al castillo de Javier (Pamplona) entre otras, acciones en pro de pueblos que han tenido una catástrofe o compañas para ayudar a pueblos desfavorecidos. Estas actividades pretenden crear un clima de colaboración y compañerismo, solidaridad y de compromiso personal.

\section{(IV) Cuarto plano: Investigación}

Finalmente, el cuarto plano se refiere a la investigación, descrito en tres aspectos: líneas prioritarias, investigación institucional, y propiamente los estudios e investigaciones, tesis y proyectos fin de carrera:

(IV-A) El primer aspecto, que se denominó Líneas prioritarias tenía como fundamento dedicar desde la universidad recursos propios para promover investigaciones sobre temas que no tienen ayuda externa pero son prioritarias para la universidad. Por ejemplo, el estudio de valores en los estudiantes a lo largo de su carrera, o temas sobre ética, o sobre el diálogo interreligioso entre otros temas, que pueden ser desarrollados desde cualquier facultad o centro universitario (como indica Eléxpuru en un documento inédito titulado Los valores de los estudiantes de la U[niversidad de] D[eusto]. Estudio comparativo por titulaciones. Promoción 2001-2005; véase también Villa y Villa, 2007).

(IV-B) El segundo aspecto en el ámbito de la investigación fue la Investigación institucional sobre temas que ayudan a recoger datos sobre el funcionamiento de la universidad y que permite tener un conocimiento sobre aspectos que interesan a la universidad, como por ejemplo: el desarrollo de valores en los estudiantes deustenses, o sus actitudes y competencias, o sobre el rendimiento universitario, solo por citar algunos temas posibles. En definitiva, se trata de tener datos que evidencien el desarrollo de los valores en el funcionamiento de la universidad.

(IV-C) El tercer aspecto fue Estudios, Tesis o Proyectos fin de carrera, realizados sobre investigaciones de carácter social en cualquier campo del saber. La revisión de las tesis doctorales registradas en la Universidad de Deusto desde 2003-2004 hasta 2008-2009 fueron de 163 defendidas de las que el número de tesis relacionadas con valores fue de 31 , lo que supone un $19 \%$. Entre los temas tratados pueden verse: violencia doméstica, separación matrimonial, responsabilidad social sobre productos, sociología del empleo y desempleo, responsabilidad social en las empresas (como indica Silvestre en un documento inédito titulado $R e$ visión de las tesis doctorales registradas en la Universidad de Deusto desde 2003-2004 hasta 2008-2009).

Estos cuatro planos, ayudan a operativizar una política de valores en la universidad, y además da lugar a tener una visión más holística sobre los valores que pretende la universidad potenciar y da un mayor sentido de identidad y congruencia, independientemente de temáticas o perspectivas desde las que se favorecen.

\section{SÍNTESIS}

El concepto de educación y enseñanza superior desarrollado en los textos claves de Kolvenbach entre 1985 y 2007 sufre una fuerte reducción hacia los inicios de la pedagogía jesuita del siglo XVI. Sin embargo, lo que parece ser una simple reformulación de la tradición jesuita contiene, al mismo tiempo, una condensación pragmática que la convierte en algo trasladable a la enseñanza práctica. 
Habría que resumir, en primer lugar, que de este modo Kolvenbach ha contribuido, esencialmente, a la transformación de los principios ledesmanianos en un esquema unitario-integral de aplicación concreta al ámbito universitario lo cual justifica, plenamente, su re-titulación como «paradigma Ledesma-Kolvenbach» del 2008. En segundo lugar, el análisis de las intervenciones claves de Kolvenbach entre 1985 y 2007 ha demostrado que la condensación pragmática ulterior de la pedagogía jesuita según Kolvenbach se efectuó mediante un cambio de referencia. A partir del 2001, el P. General holandés se aleja claramente de Newman, que había sido su referente más importante en temas de enseñanza superior desde 1987. Inspirado por los impulsos de renovación pedagógica de los jesuitas estadounidenses, se apoya en la concretización del "carisma fundacional ignaciano» realizado por parte de Ledesma mediante los "cuatro razones» de la educación y enseñanza superior jesuita. La alteración más importante que conlleva ese cambio de referencia de las reflexiones pedagógicas de Kolvenbach es la pro-

\section{NOTAS}

[1] Estos discursos del padre Peter-Hans Kolvenbach suelen recogerse en la literatura en mención a su emplazamiento geográfico y año, así la referencia de dicho autor en 1985 presente en las siguientes páginas se corresponde a [Frascatti-Roma, 1985]. Análogamente, 1987 se corresponde con [Bilbao, 1987]; 1989 con [Washington, 1989]; 1990 con [México, 1990]; 1993 con [Roma, 1993]; 1994/2008 con [Córdoba, 1994]; 2001 con [Monte-Cucco, 2001]; 2006/2008 con [Barcelona, 2006]; 2006 con [Namur, 2006] y 2007 con [Roma, 2007].

\section{BIBLIOGRAFÍA}

Agúndez Agúndez, M. (2008a). El paradigma universitario LedesmaKolvenbach. Revista de Fomento Social, 252, 63, pp. 603-631.

Agúndez Agúndez, M. (2008b). Constantes del pensamiento universitario. Peter-Hans Kolvenbach. En Agúndez Agúndez, M. (ed.) P. H. Kolvenbach S.J. Discursos universitarios. Madrid: UNIJES, pp. 9-30.

Azcarate Ristori, I. (1996). Los jesuitas en la política educativa del Ayuntamiento de Cádiz (1564-1767). Granada: Facultad de Teología.

Bailey, C. R. (1978). French secondary education 1763-1790. The secularization of ex-Jesuit colleges. American Philosophical Society.

Beirne, C. J. (1996). Jesuit education and social change in El Salvador. New York: Garland.

Biemer, G. (2009). Die Wahrheit wird stärker sein. Das Leben Kardinal Newmanns (3a ed). Francfort del Meno: Lang.

Blanchard, K. y colaboradores (2007). Liderazgo de máximo nivel. Barcelona: Gránica.

Blanco Trías, P. (1922). Valores fundamentales de la pedagogía ignaciana. Barcelona: Imprenta de Eugenio Subirana. yección de un pragmatismo realista de la educación y enseñanza superior jesuita capaz de una integración pluralista en contra de la estratificación social y sin abandonar su identidad cristiana. Ese nuevo objetivo principal reemplaza al tradicionalismo de Newman aunque la ruptura de Kolvenbach con el cardenal inglés no es completa. En la retórica sobre la «verdad total» se mantienen algunos ecos del concepto newmaniano de la universidad como guardián de una universalización anacrónica de las ciencias.

Estos ecos quedan neutralizados, finalmente, en la traslación esquemática posterior del «paradigma Ledesma-Kolvenbach» mediante su transformación unitaria-integral en un Modelo Valórico Cuatridemensional. Habría que resumir, en tercer y último lugar, que el Modelo Valórico se apoya en las alteraciones de la segunda fase de reflexiones pedagógicas de Kolvenbach entre 2001 y 2007. Su objetivo es de servir como herramienta de auto-diagnosis y de auto-reflexión de la propia Universidad con respecto al marco planteado de la educación y enseñanza superior jesuita.
Buckley, M. J. (1998). The Catholic University as Promise and Project. Reflections in a Jesuit Idiom. Washington: Georgetown University Press.

Bunes, M., Calzón, J., Elexpuru, I., Fañanás, T., Muñoz-Repiso, M. y Valle, J. (1993). Los valores en la L.O.G.S.E. Un análisis de documentos a través de la metodología de Hall-Tonna. Bilbao: Mensajero-Universidad de Deusto.

Byrnes, H. (1992). Cross-Cultural Communications: Identity in Diversity. En O'Brien, W. J. (ed.), Minding the Time 1492-1992. Jesuit Education and Issues in American Culture. Washington: Georgetown University Press, pp. 59-84.

Carmody, B. P. (1992). Conversion and Jesuit schooling in Zambia. Leiden: Brill.

Charmot, F. (1952). La pedagogía de los jesuitas. Sus principios, su actualidad. Madrid: Sapientia.

Combs, M. B. y Ruggiano, P. (eds.) (2013). Transforming ourselves, transforming the world: Justice in Jesuit Higher Education. New York: Fordham University Press. https://doi.org/10.5422/fordham/9780823254309.001.0001 
Consortium of Institutions for Development and Research in Education in Europe (1991a). Values in education in Europe collaborative programme (VEEP) proposal for the CIDREE General Assembly. La Haya.

Consortium of Institutions for Development and Research in Education in Europe (1991b). Values, Schooling and Society. La Haya.

Cuban, S. y Anderson, J. B. (2007). Where's the Justice in Service-Learning? Institutionalizing Service-Learning from a Social Justice Perspective at a Jesuit University. Equity \& Excellence in Education, 40, pp. 144-155. https://doi. org/10.1080/10665680701246609

de Dainville, F. (1940). Les jésuites et l'éducation de la société française. La naissance de l'humanisme modern. Paris: Beauchesne.

Dignath, S. (1977). Die Pädagogik der Jesuiten in den Indio-Reduktionen von Paraguay 1609-1767. Francfort del Meno: Lang.

Donnelly, F. P. (1934). Principles of Jesuit Education in Practice. Nueva York: P. J. Kenedy.

Donohue, J. W. (1963). Jesuit education. An essay on the foundations of its idea. Nueva York: Fordham University Press.

Döpfner, J. (1945). Das Verhältnis von Natur und Übernatur bei John Henry Kardinal Newmann. [Tesis doctoral inédita]. Universidad Gregoriana: Roma.

Eléxpuru Albizuri, I. y Medrano Samaniego, C. (dirs.) (2002). Desarrollo de los valores en las instituciones educativas. Bilbao: Mensajero-Universidad de Deusto. Disponible en https:// sede.educacion.gob.es/publiventa/PdfServlet?pdf=VP10784. pdf\&area $=E$

Eléxpuru, I. y Bolívar Botía, A. (Ed.) (2004). El desarrollo de valores en la universidad: experiencias y perspectivas. En Pedagogía universitaria: hacia un espacio de aprendizaje compartido. III Symposium Iberoamericano de docencia universitaria (vol. I). Bilbao: Universidad de Deusto, pp. 157-160

Friedrich, M. (2009). Theologische Einheit und soziale Kohärenz. Debatten um die Homogenität von "doctrina" im Jesuitenorden um 1600. En Büttgen, P. (ed.). Vera Doctrina. Zur Begriffsgeschichte der Lehre von Augustinus bis Descartes. Wiesbaden: Harrassowitz, pp. 297-324.

García Iglesias, L. (1994). Renovación de los colegios de jesuitas en la España contemporánea. XX Siglos, 19, pp. 104-113 y 21, pp. 93-105.

García Iglesias, L. (1995). Renovación de los colegios de jesuitas en la España contemporánea. XX Siglos, 24, pp. 63-77, 28, pp. 1930 y 29 , pp. 93-106

Gargantini, D. y Zafforoni, C. (coords.) (2009). Políticas y sistema de autoevaluación y gestión de la responsabilidad social universitaria en AUSJAL. Córdoba: Alejandría Editorial. Disponible en http:// www.ausjal.org/tl_files/ausjal/images/contenido/Investigacion/ RSU_AUSJAL\%20Version\%20Completa\%20con\%20anexos.pdf

Gómez Rodeles, C., Lecina, M., Agustí, V., Cervos, F. y Ortiz, A. (eds.) (1901). Monumenta Paedagogica Societatis lesu: Quae primam Rationem studiorum anno 1586 editam praecessere. Matriti: Typis Augustini Avrial.

González Hernández, L. (ed.) (1992). Selección de escritos del P. Peter-Hans Kolvenbach (1983-1990). Madrid: Provincia de España de la Compañía de Jesús.
Guibert, J. M. (2009). La incidencia de las universidades de la Compañía de Jesús en la cultura europea". Gregorianum, 90, 1, pp. 159-176.

Guibert, J. M. (2014). Diccionario de liderazgo ignaciano. Bilbao: Mensajero.

Hall, B. (1997). Desarrollo y gestión de valores en la universidad. Boletín de Estudios Económicos, 161, pp. 227-250.

Higher Education Quality Committee (2006). A Good Practice Guide and Self-evaluation Instruments for Managing the Quality of Service-Learning. Pretoria: The Council on Higher Education. Disponible en http://www.che.ac.za/sites/default/files/publications/HEQC_Good_Practice_guide_Jun2006_0.pdf

Hughes, T. (1981). Loyola and the Educational System of the Jesuits. Ann Arbor: Heinemann.

Hütter, R. (2013). University Education, the Unity of Knowledge and (Natural) Theology. John Henry Newman's Provocative Vision. Nova et Vetera. English Edition of the International Theological Journal, 11, pp. 1017-1056.

Kalthoff, M. A. (2001). Different Voice from the Eve of the Origin: Reconsidering John Henry Newman on Christianity, Science, and Intelligent Design. Perspectives on Science and Christian Faith, 53, pp. 14-23.

Kolvenbach, P. H. (1985/2008). La Universidad Jesuítica hoy. En Agúndez Agúndez, M. (ed.). Kolvenbach, P. H. Discursos universitarios. Madrid: UNIJES, pp. 31-43.

Kolvenbach, P. H. (1986/1992). Sobre las «características» de la educación jesuítica. En González Hernández, L. (ed.). Selección de escritos del P. Peter-Hans Kolvenbach (1983-1990). Madrid: Provincia de España de la Compañía de Jesús, pp. 143-145.

Kolvenbach, P. H. (1987/2008). En el centenario de la Universidad de Deusto. En Agúndez Agúndez, M. (ed.). Kolvenbach, P. H. Discursos universitarios. Madrid: UNIJES, pp. 44-54.

Kolvenbach, P. H. (1989/2008). Características de nuestra educación. En Agúndez Agúndez, M. (ed.). Kolvenbach, P. H. Discursos universitarios. Madrid: UNIJES, pp. 55-73.

Kolvenbach, P. H. (1990/2008). Un nuevo modelo de universidad. En Agúndez Agúndez, M. (ed.). Kolvenbach, P. H. Discursos universitarios. Madrid: UNIJES, pp. 87-98.

Kolvenbach, P. H. (1991). Características de las Instituciones de Enseñanza Superior de los Jesuitas. Córdoba: ETEA.

Kolvenbach, P. H. (1993/2008). La Pedagogía Ignaciana Hoy. En Agúndez Agúndez, M. (ed.). Kolvenbach, P. H. Discursos universitarios. Madrid: UNIJES, pp. 124-139.

Kolvenbach, P. H. (1994). Discours aux enseignants (Erpent, 1991). En Les collèges jésuites d'hier à demain. Pédagogie et spiritualité. Bruselas: Lumen Vitae, pp. 133-148.

Kolvenbach, P. H. (1994/2008). Presente y futuro de INSA-ETEA. En Agúndez Agúndez, M. (ed.). Kolvenbach, P. H. Discursos universitarios. Madrid: UNIJES, pp. 140-153.

Kolvenbach, P. H. (2001/2008). La Universidad de la Compañía de Jesús a la luz del carisma Ignaciano. En Agúndez Agúndez, M. (ed.). Kolvenbach, P. H. Discursos universitarios. Madrid: UNIJES, pp. 190-209. 
Kolvenbach, P. H. (2006/2008a). Evolución del encuentro entre Ciencia y Fe. En Agúndez Agúndez, M. (ed.). Kolvenbach, P. H. Discursos universitarios. Madrid: UNIJES, pp. 236-248.

Kolvenbach, P. H. (2006/2008b). A las Facultades Universitarias de Nôtre Dame de la Paix. En Agúndez Agúndez, M. (ed.). Kolvenbach, P. H. Discursos universitarios. Madrid: UNIJES, pp. 249-255.

Kolvenbach, P. H. (2007/2008). Al Consejo directivo de la Universidad de Georgetown. En Kolvenbach, P. H. Discursos universitarios. Madrid: UNIJES, pp. 256-266.

Labrador Herraiz, C. (1987) El sistema educativo de la Compañía de Jesús. Continuidad e innovación: ante el cuarto centenario de la Ratio Studiorum, Madrid: Universidad Pontificia de Comillas.

Labrador Herraiz, C. (1999). Estudio histórico-pedagógico. En Gil Coria, E. (ed.), La Pedagogía de los Jesuitas, ayer y hoy. Madrid: Universidad Pontificia Comillas, pp. 23-56.

Lara, J. (2010). La persona humana como sujeto de la educación (antropología zubiriana). En Guibert, J. M. (coord.). Cooperativismo, empresa y universidad. In memoriam de Dionisio Aranzadi Tellería S J. Bilbao: Universidad de Deusto, pp. 393-418.

Larrañaga Oyarzabal, X. (2012). Jesucristo en nosotros. La doctrina de la justificación en John Henry Newman. Salamanca: Universidad Pontificia de Salamanca.

Ledesma, D. (1611). Catecismo, y Declaración breve de la Doctrina Christiana, por preguntas y respuestas entre Maestro y Discípulo. Sevilla: Alonso Gamarra.

Lemke Duque, C. A. (2014). Europabild - Kulturwissenschaften Staatsbegriff. Die Revista de Occidente (1923-1936) und der deutsch-spanische Kulturtransfer der Zwischenkriegszeit. Francfort del Meno: Vervuert.

Lowney, C. (2005). El liderazgo al estilo de los jesuitas. Las mejores prácticas de una compañía de 450 años que cambió el mundo. Barcelona: Norma.

Malaxechevarría, J. (1926). La Compañía de Jesús por la instrucción del pueblo vasco en los siglos XVII y XVIII. Ensayo histórico. Donostia-San Sebastián: Imprenta y Librería San Ignacio de Loyola.

McDermott, I. y O’Connor, J. (1999). Programación neurolingüística para directivos. Barcelona: Urano.

McKevitt, G. (2008). Jesuit Schools in the USA 1814-1970. En Worcester, T. (ed.). The Cambridge companion to the Jesuits. Cambridge: Cambridge University Press, pp. 278-297. https://doi. org/10.1017/CCOL9780521857314.017

Muñoz-Repiso, M. (1994). Valores y autonomía del centro docente. En Villa, A. (ed.). Autonomía institucional de los centros educativos. Presupuestos, organización y estrategias. Bilbao: Universidad de Deusto, pp. 351-364.

Newmann, J. H. (1873). Letter to J. Walker of Scarborough on Darwin's theory of evolution, May 22, 1868. En Dessain, C. S. y Gornal, T. (eds.). The Letters and Diaries of John Henry Newman (vol. XXIV). Oxford: Clarendon Press, pp. 77-78.

Newmann, J. H. (1885/1907). The Idea of a University defined and illustrated. En Nine Discourses delivered to Catholics in Dublin in occasional lectures and essays addressed to the members of the Catholic University. Londres: Longmans.
Oráa, J. (2010). Prólogo. En Guibert, J. M. (ed.) (2010). Cooperativismo, Empresa y Universidad. In memoriam de Dionisio Aranzadi Tellería S J. Bilbao: Universidad de Deusto, pp. 13-14.

Organization for Economic Co-operation and Development (1993). The curriculum redefined. Conference Schooling for the 21st. Century. Paris: Organization for Economic Co-operation and Development.

Padberg, J. W. (1992). Who are these people and what are they doing and why. Conversations on Jesuit Higher Education, 1, pp. 1-3.

Padberg, J. W. (2000). Development of the Ratio Studiorum. En Duminuco, V. J. (ed.). The Jesuit Ratio Studiorum: 400th Anniversary perspectives. Nueva York: Fordham University Press, pp. 80-100.

Rehg, W. (2006). Epilogue: Do Jesuit Scholary Endeavors Cohere? Self-Reckoning and the Postmodern Challenge. En Clooney, F. X. (ed.), Jesuit postmodern. Scholarship, vocation, and identity in the 21st century. Oxford: Lexington Books, (pp. 195-218).

Revuelta González, M. (1998). Los colegios de jesuitas y su tradición educativa (1868-1906). Madrid: Universidad Pontificia de Comillas.

Resines Llorente, L. (1997). El catecismo de Diego de Ledesma. Archivum Historicum Societatis lesu, 66, pp. 249-274.

Ritzler, B. (2000). Freiheit in der Umarmung des ewig Liebenden. Die historische Entwicklung des Personenverständnisses bei Jacques Maritain. Berna: Lang.

Schneck, S. (2002) (ed.). Scheler's Acting Persons: New Perspectives. Nueva York: Rodopi.

Schwanke, J. (2011). John Henry Newmans Konversion. Sein Weg zur katholischen Kirche aus protestantischer Sicht. Berlin: De Gruyter. https://doi.org/10.1515/9783110228939

Schwanke, J. (2013). Die Universitätsidee des John Henry Newman eine Vergegenwärtigung". En J. Schwanke, J. (ed.). Bildung durch Wissenschaft - wie ist das zu verstehen? Die Idee der Universität in ihrer Geschichte. Berlin: Bund Freiheit der Wissenschaft, pp. 50-61.

Schwartz, S. H. (2001). ¿Existen aspectos universales en la estructura y contenido de los valores humanos? En Ros, M. y Gouveia, V. V. (coords.), Psicología de los valores humanos. Madrid: Biblioteca Nueva, pp. 53-99.

Spader, P. H. (2002). Scheler's Ethical Personalism. Its Logic, Development, and Promise. Nueva York: Fordham University Press. https://doi.org/10.5422/fso/9780823221776.001.0001

Whitehead, M. (2013). English Jesuit education. Expulsion, suppression, survival and restoration 1762-1803. Farnham: Ashgate.

Villa, A. y Villa, O. (2007). El aprendizaje basado en competencias y el desarrollo de la dimensión social en las universidades. Educar, 40 , pp. $15-48$.

\section{Recursos de Internet}

Características de la Educación de la Compañía de Jesús. [En línea]. Disponible en http://www.flacsi.net/wp-content/ uploads/2011/11/Caracteristicas-de-la-Educacion-de-laS.J.-1986.pdf

Duminuco, V. J. Prólogo. En Pedagogía Ignaciana. Un planteamiento práctico. Disponible en http://www.google.es/url? $s a=t \& r c t=j \& q=\& e s r c=s \&$ source $=w e b \& c d=\& v e d=0 a h U K E w j$ 5rOXGvN_RAhUBJcAKHdW8AhQQFgghMAA\&url=http\%3A 
\%2F\%2Fwww.flacsi.net\%2Fwp-content\%2Fuploads\%2F201 1\%2F11\%2FPedagog\%25C3\%25ADa-Ignaciana-Un-planteamiento-pr\%25C3\%25A1ctico.doc\&usg=AFQjCNGTiCvNDWTWn OfcGJAlu7nGR4m3Hw\&bvm=bv.145063293,d.ZGg

Nicolás Pachón, A. (2008). Misión y Universidad. ¿Qué futuro queremos? [En línea]. Disponible en http://www.ausjal.org/documentos-padre-general.htm

Nicolás Pachón, A. (2010a). Profundidad, universalidad y ministerio académico. Desafíos a la educación superior jesuita de hoy. [En línea]. Disponible en http://www.ausjal.org/documentospadre-general.html

Nicolás Pachón, A. (2010b). Depth, Universality, and Learned Ministry. Challenges to Jesuit higher education today. [En línea]. Disponible en http://www.ausjal.org/documentos-padre-general.html

Nicolás Pachón, A. (2011). Lección inaugural del curso académico 2011-2012 en la Universidad de Deusto. [En línea]. Disponible en http://www.ausjal.org/documentos-padre-general.html
Nicolás Pachón, A. (2013a). Sobre el liderazgo ignaciano. [En línea]. Disponible en http://www.ausjal.org/documentos-padre-general.html

Nicolás Pachón, A. (2013b). Sobre la Misión universitaria de la Compañía. [En línea]. Disponible en http://www.ausjal.org/documentos-padre-general.html

Nicolás Pachón, A. (2013c). La Educación en la Compañía de Jesús. [En línea]. Disponible en http://www.ausjal.org/documentospadre-general.html

Nicolás Pachón, A. (2013d). En busca de líderes espirituales (2013). [En línea]. Disponible en http://www.ausjal.org/documentospadre-general.html

Seminario sobre Liderazgo Ignaciano y Justicia Social. [En línea]. Disponible en http://www.ausjal.org/multimedia.html 